\title{
Optimizing Surgical Results from Facial Trauma Through a Facial Basal Registration
}

\author{
Adaia Valls ${ }^{1}$, Gloria Molins ${ }^{2 *}$, Jorge Masiá ${ }^{3}$ and FedericoHernández-Alfaro ${ }^{3}$ \\ ${ }^{1}$ Instituto Maxilofacial-Centro Médico Teknon, Spain \\ ${ }^{2}$ Centro Médico Teknon-Anestalia, Spain \\ ${ }^{3}$ Instituto Maxilofacial-Centro Médico Teknon, Spain \\ *Corresponding author: Gloria Molins, Centro Médico Teknon-Anestalia, c/Vilana, 12, Barcelona, 08017, Spain
}

Submission: 眥 November 09, 2018; Published: 眥 December 18, 2018

\begin{abstract}
Summary
Surgery is the treatment of choice for most of maxillofacial fractures (MFF). Hence, three-dimensional (3D) virtual planning with computer software technologies and navigation assisted surgery has emerged as an integral tool through the mirroring hemifaces technique. However, the aforementioned protocol is not advisable for asymmetric faces nor is it applicable for bilateral MFF. Therefore, the aim of this paper is to describe an innovative workflow based on keeping a prior 3D registry of the healthy patient or so called "facial print".
\end{abstract}

This protocol includes a scan of the patient, surface intraoral scanning of both dental arches and photographs, and the subsequent fusion of the three data sets. Twenty subjects who were interested in having a "facial print" were enrolled in the trial. The studied sample comprised of 12 women and 8 men with a mean age of 35.7 years. Total patient registration time for the three records was on average 13 minutes and the matching work for "virtual patient" generation took another 15 minutes on average.

In conclusion, this innovative workflow has the potential to improve surgical accuracy and predictability, reduce operative time and level up surgeon's comfort.

Keywords: Computer assisted surgery; Facial reconstruction; Facial-print; CBCT; Surface intraoral scanning; 3D virtual planning

\section{Introduction}

Maxillofacial fractures (MFF) account for $24 \%$ of all trauma injuries and can occur either as an isolated injury or in combination with other severe injuries, including cranial, spinal, and upper and lower body injuries [1]. The literature on facial trauma epidemiology varies widely, because it is constantly influenced by the geography, socioeconomic status, and cultural differences. Overall, however, road traffic accidents are the most prevalent aetiology, followed in decreasing rank order by assault, sports, falls and occupational hazards. The highest incidence of MFF is observed in men between 20 and 29 years old, although older women (those over 65 years old) are more likely than older men to sustain a MFF [1-3]. The most commonly fractured bone is the nose, followed in descending order by the mandible, the zygomatic complex, and then midface and orbital fractures [4].

Surgery is the treatment of choice for most patients with MFF. The face is a three-dimensional (3D) structure and plays an important role in socialization; so an optimal outcome is of the upmost importance when managing MFF. For this reason, 3D virtual planning with computer software technologies and navigation assisted surgery $[5,6]$ has emerged as an integral tool to achieve optimal outcomes. Such cutting-edge technologies are very helpful in the surgical planning and treatment of patients with unilateral trauma of the facial skeleton, because bone from the uninjured hemiface can then be mirrored and repositioned on the contralateral hemiface using a registration technique, thereby generating a target position for the realignment of any displaced bony fragments on the injured side of the face [6]. Nevertheless, several studies have demonstrated and quantified the asymmetries in normal and uninjured faces $[7,8]$, so the aforementioned protocol is not advisable for assessing asymmetric faces and neither is it applicable to bilateral MFF.

In other words, we have not yet reached the "gold standard" technique for MFF management. Therefore, the aim of this paper is to propose an innovative workflow to improve the handling of MFF, which is based on keeping a prior registry of healthy patients or so called "facial-prints" that includes the facial soft tissue mask, and the underlying bone and teeth. The "virtual patient", thus created, will be the reference model for excellent 3D virtual planning in case the patient suffers a future MFF. By doing so, we can overcome the aforementioned concerns related to facial asymmetries, mirroring discrepancies or panfacial fractures.

\section{Materials and Methods}

A prospective study was carried out in a group of 20 consecutive patients who were interested in having a "facial-print" and vol- 
unteered to take part in the trial. Informed consent was obtained from all individual participants included in the study. The proposed workflow only requires a single cone beam computed tomography (CBCT) scan of the patient, surface intraoral scanning of both dental arches and two-dimensional (2D) photographs, and the subsequent fusion of the three sets of data.

A low radiation CBCT is performed in an i-CAT scanner (Imaging Sciences International, Inc., Hatfield, USA) with the patient breathing quietly, sitting upright in natural head position, the tongue is in a relaxed position, and the mandible is in centric relation with a $2 \mathrm{~mm}$ wax bite in place in order to avoid direct tooth contact (Figure 1). CBCT's are collected in DICOM format (Dental Imaging
Communication) and exported to a specific software (Dolphin ${ }^{\circledR}$ 3D Orthognathic Surgery Planning Software Version 11.8, Chatsworth, California, USA), in which DICOM files are segmented and processed in order to obtain a 'clean' 3D representation, which is then stored as an STL file. Afterwards, surface scanning of both dental arches is achieved with the Lava Scan ST scanner (3M ESPE, Ann Arbor, MI, USA), thereby producing another STL file (Figure 2). Finally, a frontal 2D photograph in resting position is taken (Figure 3). The three STL files are fused (Figure 4) using the Dolphin ${ }^{\circledR}$ software with a "best fit" algorithm. The system uses surface-based rigid registration using ICP (iterative closest point) in order to minimize rotational and translational differences between the three datasets (Figure 5).

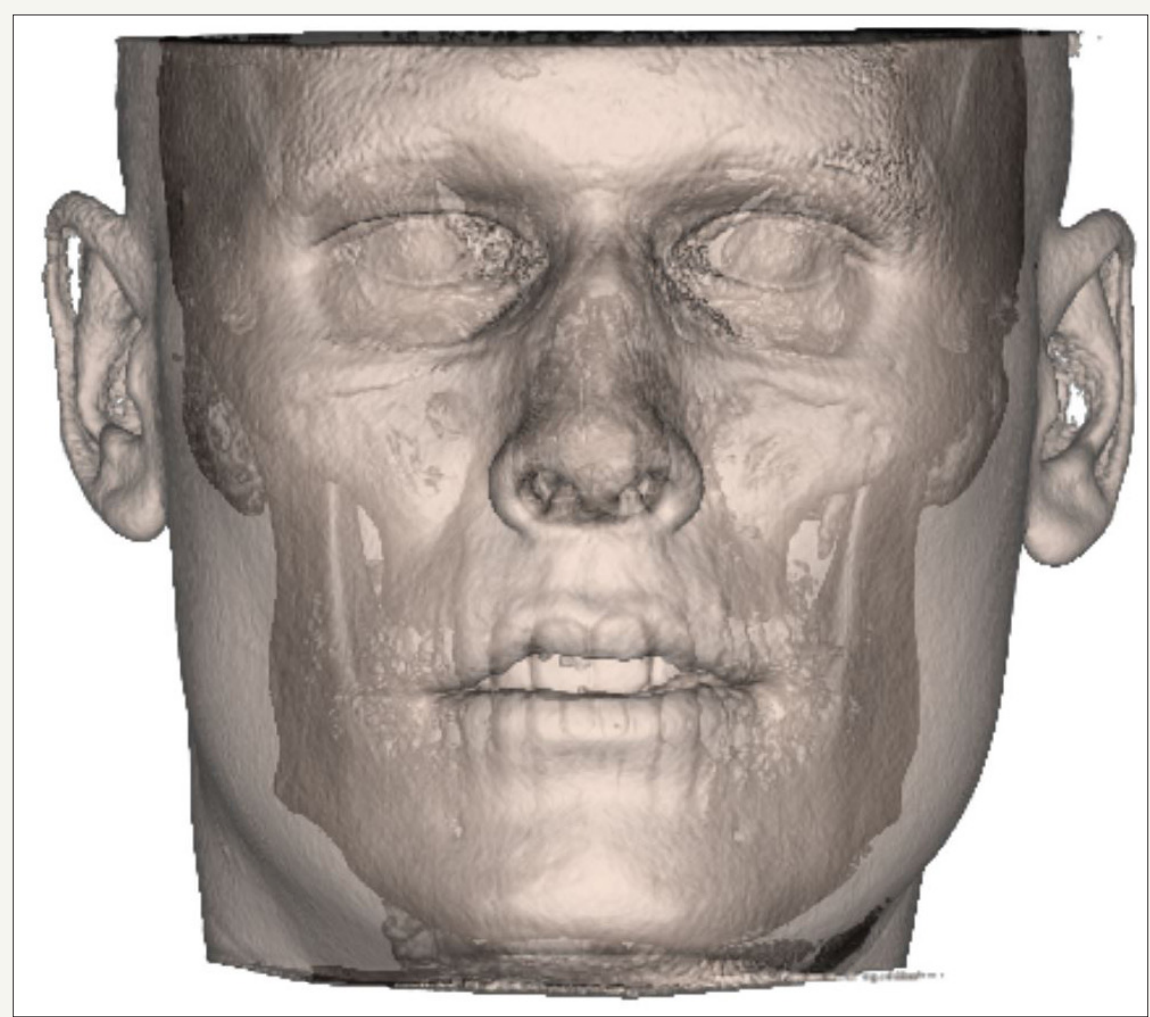

Figure 1: A CBCT scan is taken with the patient breathing quietly, sitting upright in natural head position, the tongue is in a relaxed position, and the mandible is in centric relation with a $2 \mathrm{~mm}$ wax bite in place in order to avoid direct tooth contact.

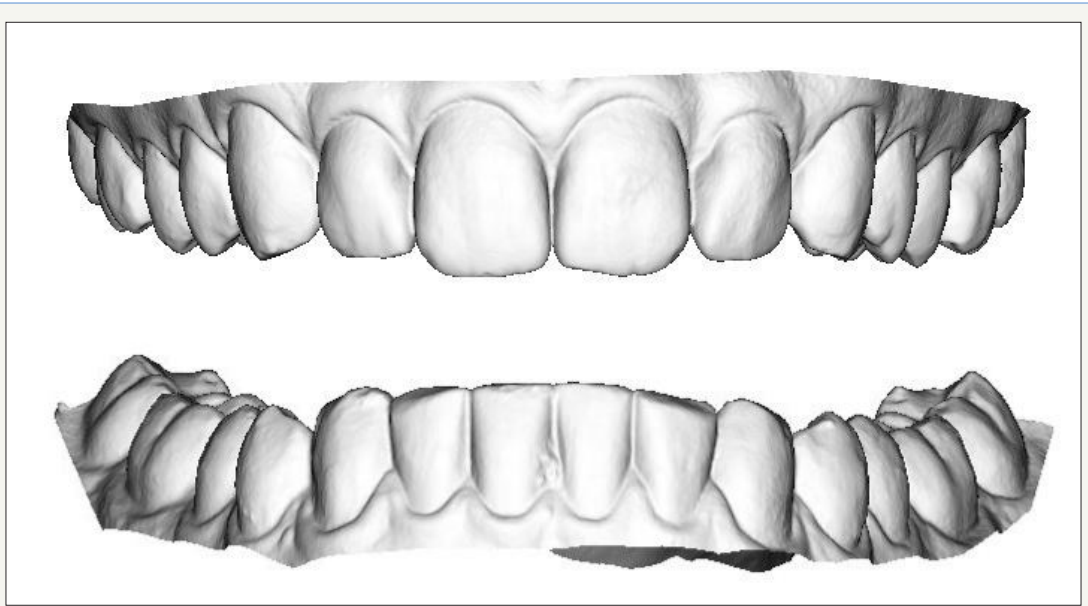

Figure 2: Surface intraoral scanning of both dental arches. 


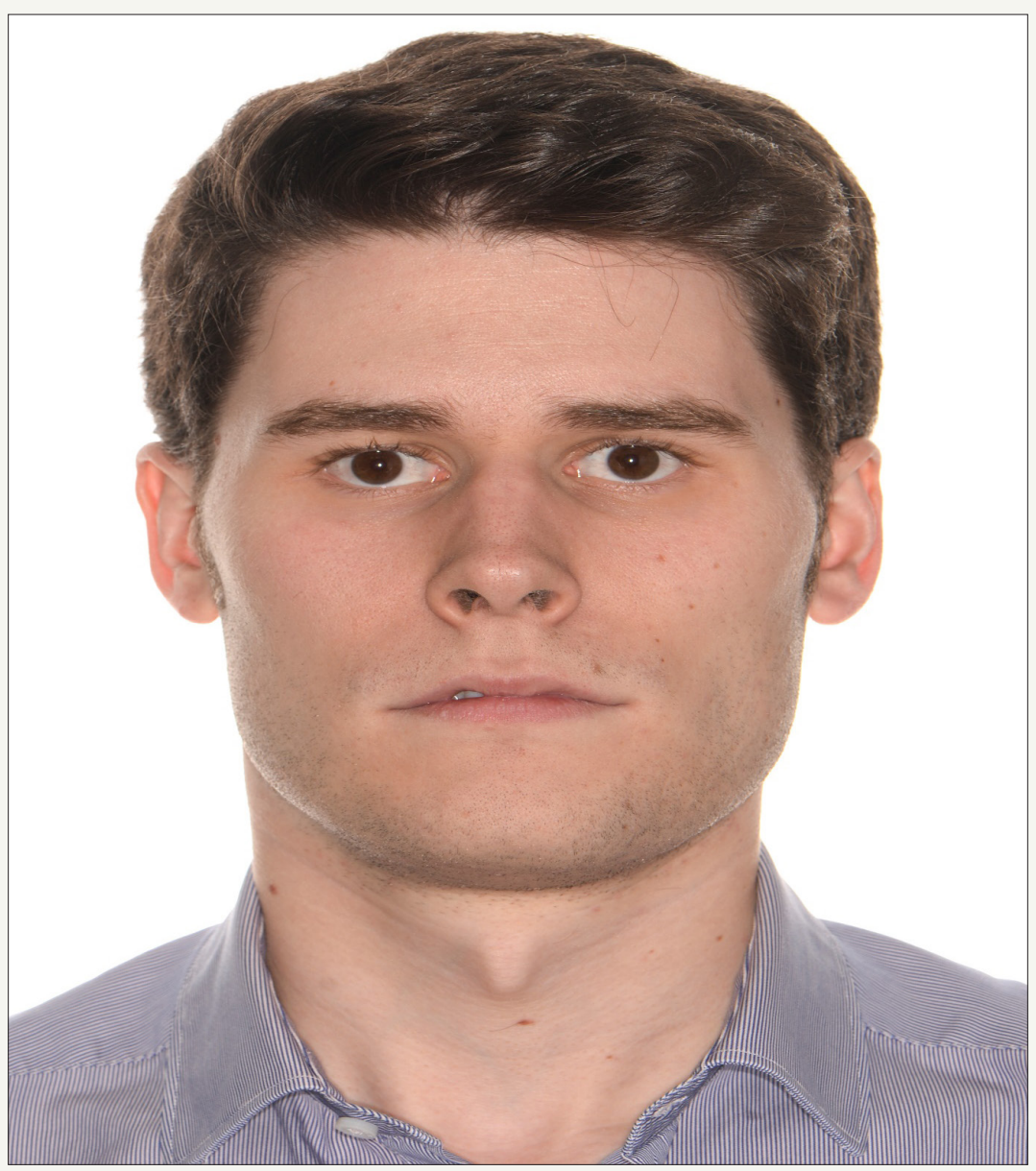

Figure 3: Frontal 2D photograph in resting position.

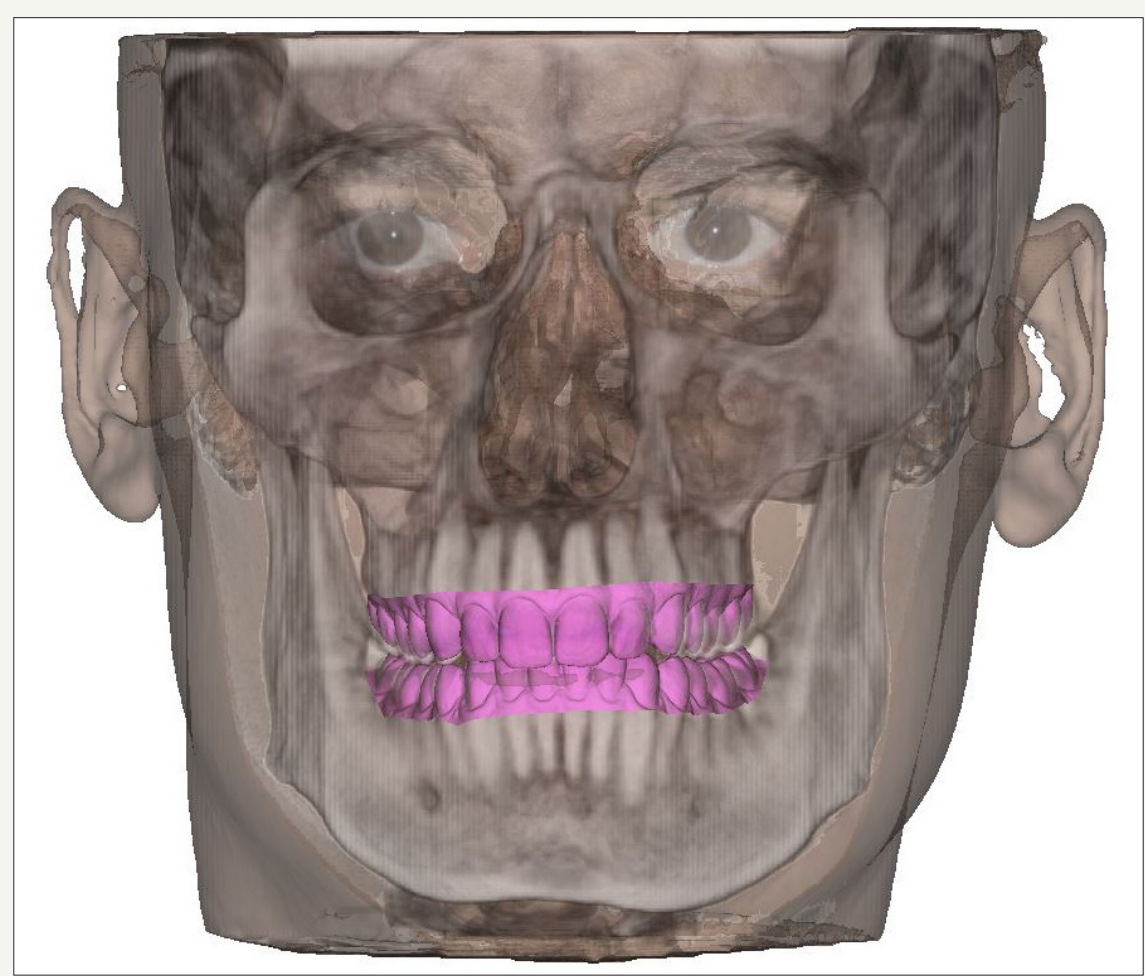

Figure 4: "Facial-print" or "virtual patient". 


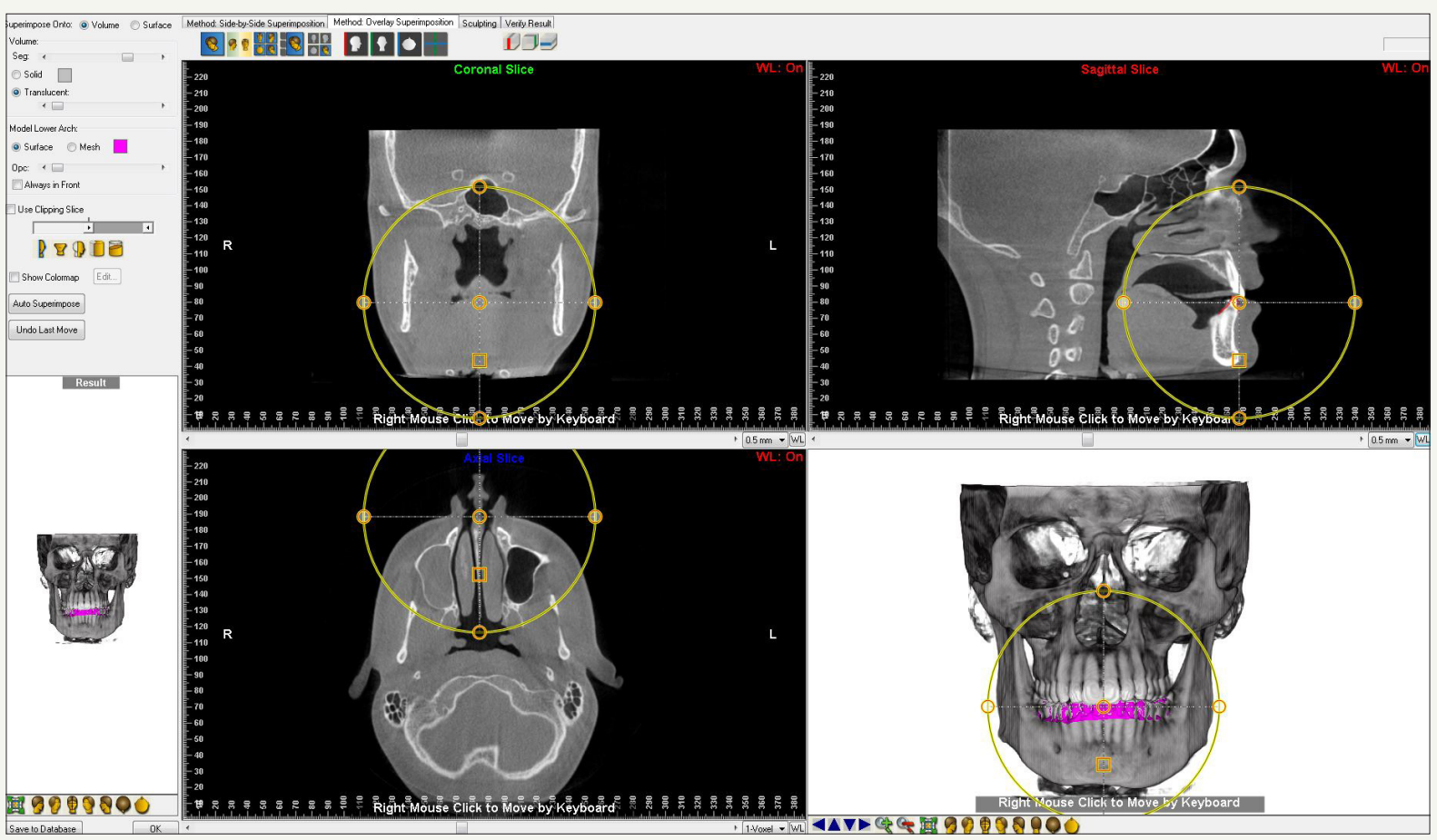

Figure 5: Fusing work using Dolphin ${ }^{\circledR}$ software.

The implementation of this protocol at our centre started up with a trial period in order to assess the ability of our healthcare team to carry out the exams and the fusing work properly. Two physician assistants (IFC and NLT), with high level of experience with 3D surgery planning software, generated all virtual models independently on two separate occasions (two weeks apart), thus avoiding inter and intra-operator differences respectively.

\section{Results}

Twenty subjects who were interested in having a "facialprint" were enrolled in the trial. The studied sample comprised 12 women (60\%) and 8 men (40\%) with a mean age of 35.7 years (range 18-67). Total patient registration time for the three records was 13 minutes on average (range 11-15 minutes) (specifically 2 minutes for CBCT scan, 3 minutes for facial pictures and 8 minutes for surface intraoral scanning of both dental arches). Fusing work for virtual patient generation took an additional15 minutes on average (range 13-16 minutes).

\section{Discussion}

In an effort to improve diagnosis and outcomes for the treatment of MFF, we have created a database with facial virtual anatomic models of every single patient who requests it. This "facial-print" is handed to the patient via USB stick, in the case they require it abroad or if they simply want to get operated on at another medical centre. To restore aesthetics and function are the major cornerstones when dealing with MFF, which is why not only the skeleton is recorded in the "facial-print" through a low radiation CBCT scan [9]. On the one hand, 2D pictures are taken because facial soft tissue texture and appearance are crucial to achieve aesthetic results. On the other hand, the occlusion is also mandatory for the functional aspect. Therefore, the surface intraoral scanning of both dental arches is required because CBCT scans produce inaccurate visualization of the inter-occlusal relationship $[10,11]$.

The generated virtual "facial-print" not only overcomes troubles related to facial asymmetries or panfacial fractures, but also facilitates the manufacture of well-tailored physical elements such as surgical splints, cutting templates or anatomical prostheses. Furthermore, this process can also be used for other kinds of facial reconstructive surgeries, such as oncologic reconstructions. This methodology is considered a key tool in modern 3D virtual planning surgery, so it is mainly recommended for surgeons with high levels of experience with computer software technologies and, if possible, with navigation assisted surgery.

\section{Conclusion}

In conclusion, this innovative workflow based on a prior "facial-print" record that includes the facial soft tissue mask and underlying bone and teeth; can be considered a key tool in 3D virtual planning of MFF surgeries. It has the potential to improve surgical accuracy and predictability, reduce operative time, level up surgeon's comfort and therefore, to improve the final outcome.

\section{Ethical Approval}

All procedures performed in studies involving human participants were in accordance with the ethical standards of the institutional and/or national research committee and with the 1964 Helsinki declaration and its later amendments or comparable ethical standards.

\section{Acknowledgement}

The authors gratefully acknowledge every staff member at the Institute of Maxillofacial Surgery, Teknon Medical Centre Barcelona, for their good contributions. 


\section{References}

1. Manodh P, Prabhu Shankar D, Pradeep D, Santhosh R, Murugan A (2016) Incidence and patterns of maxillofacial trauma-a retrospective analysis of 3611 patients-an updates. Oral Maxillofac Surg 20(4): 377-383.

2. de Lucena AL, da Silva Filho GF, de Almeida Pinto Sarmento TC, de Carvalho SH, Fonseca FR, et al. (2016) Epidemiological profile of facial fractures and their relationship with clinical-epidemiological variables. J Craniofac Surg 27(2): 345-349.

3. Atisha DM, Burr T, Allori AC, Puscas L, Erdmann D, et al. (2016) Facial fractures in the aging population. Plast Reconstr Surg 137(2): 587-593.

4. Al Shetawi AH, Lim CA, Singh YK, Portnof JE, Blumberg SM (2016) Pediatric maxillofacial trauma: a review of 156 patients. J Oral Maxillofac Surg 74(7): 1420.

5. Shin HS, Kim SY, Cha HG, Han BL, Nam SM (2016) Real time navigationassisted orbital wall reconstruction in blowout fractures. J Craniofac Surg 27(2): 370-373

6. Baumann A, Sinko K, Dorner G (2015) Late reconstruction of the orbit with patient-specific implants using computer-aided planning and navigation. J Oral Maxillofac Surg 73(12): S101-S106.
7. Belcastro A, Willing R, Jenkyn T, Johnson M, Galil K, et al. (2016) A threedimensional analysis of zygomatic symmetry in normal, uninjured faces. J Craniofac Surg 27(2): 504-508.

8. Sidequersky FV, Mapelli A, Annoni I, Zago M, De Felício CM, et al. (2016) Three-dimensional motion analysis of facial movement during verbal and nonverbal expressions in healthy subjects. Clin Anat 29(8): 991-997.

9. Feragalli B, Rampado O, Abate C, Macrì M, Festa F, et al. (2017) Cone beam computed tomography for dental and maxillofacial imaging: technique improvement and low-dose protocols. Radiol Med 122(8): 581-588.

10. Aboul-Hosn Centenero S, Hernández-Alfaro F (2012) 3D planning in orthognathic surgery: CAD/CAM surgical splints and prediction of the soft and hard tissues results - our experience in 16 cases. J Craniomaxillofac Surg 40(2): 162-168.

11. Hernández-Alfaro F, Guijarro-Martínez R (2013) New protocol for three-dimensional surgical planning and CAD/CAM splint generation in orthognathic surgery: an in vitro and in vivo study. Int J Oral Maxillofac Surg 42(12): 1547-1556.

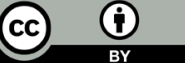

Creative Commons Attribution 4.0 International License

For possible submissions Click Here

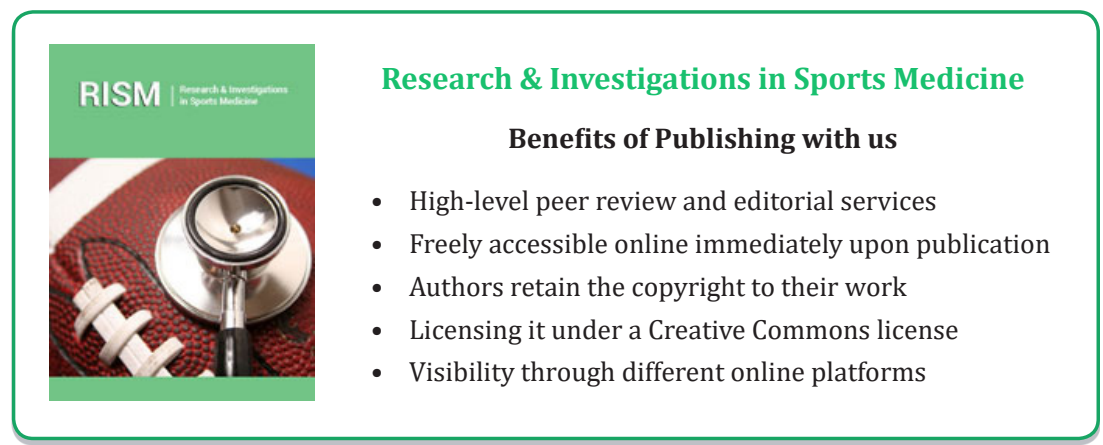

